\title{
Aprendizaje basado en proyectos en el Grado en ingeniería de la energía
}

\author{
Isabel Martón ${ }^{\mathrm{a}}$, Sergio Gallardo ${ }^{\mathrm{b}}$, José Felipe Villanueva ${ }^{\mathrm{c}}$, Sofia Carlos Alberola ${ }^{\mathrm{d}}$, Ana \\ Sánchez ${ }^{\mathrm{e}}$
}

apto. de Estadística e Investigación Operativa Aplicadas y Calidad, Universitat Politècnica de València, Spain, email: ismarllu@,upvnet.upv.es; ${ }^{b} D$ pto. de Ingeniería Química y Nuclear, Universitat Politècnica de València, Spain, email: sergalbe@iqn.upv.es; ' Dpto. de Ingeniería Química y Nuclear, Universitat Politècnica de València, Spain, email: jovillo0@upvnet.upv.es; ${ }^{\mathrm{d} D p t o . ~ d e ~ I n g e n i e r i ́ a ~}$ Química y Nuclear, Universitat Politècnica de València, Spain, email: scarlos@iqn.upv.es; ${ }^{e}$ Dpto. de Estadística e Investigación Operativa Aplicadas y Calidad, Universitat Politècnica de València, Spain, email: aisanche@eio.upv.es

\begin{abstract}
In the standards and guidelines for quality assurance in the European Higher Education Area (EHEA), is stablished that one of the keys to combine the knowledge and skills of the learners is the implementation of new pedagogical active methodologies which are more student-centred, and learning based on competences. One of the most widely used and effective active learning methodologies is the Project Based Learning (PBL). In recent decades, a significant number of initiatives have been carried out, but, in most cases, they are limited to implementations in individual subjects, with no scope in the degree curriculum. In this frame, the main goal of this work is the development of the project-based learning in two subjects of the bachelor's degree in Energy Engineering in the Universitat Politècnica de València. The same problem will be analyzed from the practical point of view making use of both theorical concepts of the subject "Statistic" and their subsequent implementation within the subject "Energy and Sustainable Development". Thus, this paper presents the developed methodology, the coordination between these two subjects and how it has been implemented, the achieved results and the initial conclusions obtained.
\end{abstract}

Keywords: Project Based Learning (PBL), Energy, Engineering, Statistic

\section{Resumen}

En el marco del Espacio Europeo de Educación Superior (EEES) se considera entre sus directrices el aprendizaje por competencias, la enseñanza centrada en el estudiante y el aprendizaje activo Una de las metodologías de aprendizaje activo más utilizadas y efectivas es el Aprendizaje Basado en Proyectos (ABP). En las últimas décadas, se han llevado a cabo un número significativo de iniciativas, pero, en la mayoría de los casos, se limitan a implementaciones en asignaturas individuales, sin alcance en el currículo del 
grado. En este marco, el objetivo principal de este trabajo es el desarrollo del aprendizaje basado en proyectos en dos asignaturas del Grado en Ingeniería de la Energía en la Universitat Politècnica de València. El mismo problema se analizará desde el punto de vista práctico, haciendo uso de los dos conceptos teóricos de la asignatura "Estadística" y su posterior implementación dentro de la asignatura "Energía y desarrollo sostenible". El objetivo del presente trabajo es mostrar la metodología desarrollada, cómo se ha implementado, los resultados obtenidos y las conclusiones iniciales obtenidas.

Palabras clave: Aprendizaje Basado en Proyectos (ABP), Energía, Ingeniería, Estadística

\section{Introducción}

El marco del Espacio Europeo de Educación Superior (EEES) considera entre sus directrices el aprendizaje por competencias, la enseñanza centrada en el estudiante y el aprendizaje activo (Garcia, 2017). Entre las metodologías de aprendizaje activo tiene un lugar singular el aprendizaje basado en proyectos (Project Based Learning (PBL)). El PBL, es una metodología pedagógica práctica, en la que se propone como objetivo que el alumno desarrolle un proyecto en el cual investigar y resolver un problema real aplicando los conceptos teóricos desde un punto de vista práctico. Es una metodología muy versátil que permite adaptar según sea el objetivo en cada caso, tanto el alcance el aprendizaje, como el grado de interdisciplinaridad y profesionalidad de éste.

La integración del PBL en el proceso de aprendizaje ha demostrado su eficacia para trabajar conjuntamente competencias específicas y competencias transversales de la asignatura o asignaturas a las que aplique, así como para preparar al estudiante para su futuro desarrollo profesional (Huff, 2016). En este sentido se han desarrollado estudios en los que se presentan metodologías para evaluar estas competencias y la valoración tanto como docentes como alumnos. En (Cuiñas, 2016) se presenta el procedimiento de evaluación de competencias en un máster en la Universidad de Vigo, en el que intervienen tanto docentes como alumnos siguiendo unas rúbricas conocidas y publicadas. En (Navarro, 2015) también se presentan rúbricas para evaluar competencias mostrando los resultados de su aplicación a un grupo experimental en un grado de la Universidad de Alicante. De estos resultados se desprende que la metodología docente aplicada tiene efectos positivos sobre el rendimiento académico y la motivación del alumnado.

La aplicación de esta metodología requiere por parte del profesor el diseño y elaboración de un programa con las actividades que los estudiantes deberán llevar a cabo, tanto de forma presencial como no presencial. En cuanto a la evaluación, permite realizar un adecuado seguimiento del grado de consecución de los objetivos formativos planteados, en términos de las habilidades y competencias adquiridas por parte de los alumnos. Asimismo, este giro metodológico precisa de un cambio en el rol del profesor, el cual debe asumir funciones de guía y acompañante del alumnado en el proceso de aprendizaje (Marimon, 2013). 
En las últimas décadas se ha llevado a cabo un número importante de iniciativas, pero, en la mayoría de los casos, se limitan a implementaciones en asignaturas individuales, sin alcance en el currículo de las titulaciones.

En el caso del Grado de Ingeniero de la Energía (GIE), de la Univesitat Politècnica de València (UPV), los autores del presente trabajo han detectado en diversas asignaturas carencias en conceptos básicos que dificultan el óptimo desarrollo de ciertas competencias específicas. En este marco, el departamento de Ingeniería Química y Nuclear (DIQN) y el departamento de Estadística e Investigación Operativa Aplicadas y Calidad (DEIOAC) han trabajado conjuntamente para identificar debilidades, amenazas, fortalezas y oportunidades en algunas asignaturas de GIE y se ha podido constatar que en la asignatura de primer curso "Estadística" y en la asignatura de segundo curso "Energía y Desarrollo Sostenible" existe una clara oportunidad de mejora en el desempeño docente. Para ello, se proponer optimizar los recursos didácticos fomentando las sinergias entre las dos asignaturas. Más concretamente, el problema se ha puesto de manifiesto al tratar de aplicar técnicas estadísticas a problemas relacionados con la generación de energía. En el curso 2017/2018, se determinó que la dificultad en seguir los problemas propuestos y resueltos en clase eran más acusados en el tema de producción de energía eólica, ya que para dicho cálculo se parte de una distribución estadística que caracteriza el comportamiento de la velocidad del viento en frecuencia, función de distribución Weibull. Por otra parte, también se ha constatado en los últimos años, que el alumnado se siente especialmente motivado en temas de energías renovables (como la eólica). Aunando estos dos hechos, se han podido identificar una debilidad (carencias de base teórica y técnica) y una fortaleza (atracción por las energías renovables) en las asignaturas mencionadas anteriormente.

En definitiva, la coordinación vertical ha permitido detectar esta debilidad y fortaleza, permitiendo establecer una estrategia conjunta de enseñanza-aprendizaje que vertebre las dos asignaturas.

\section{Objetivos}

El objetivo general del trabajo es el de diseñar actividades enfocadas al aprendizaje basado en proyectos $\mathrm{y}$, desarrollar y establecer metodologías, definir técnicas de evaluación apropiadas de evaluación centradas en el aprendizaje basado en proyectos en la asignatura de "Energía y Desarrollo Sostenible", potenciando el uso de los conceptos aprendidos en la asignatura de "Estadística" en el Grado de Ingeniero de la Energía.

Este trabajo se enmarca en un Proyecto de Innovación y Mejora Educativa de la Universidat Politècnica de València de dos años de duración (2018-2020) aplicado al Grado de Ingeniero de la Energía (GIE). Como el proyecto está en el primer año de ejecución, se van a mostrar la metodología y resultados obtenidos durante este periodo, durante el primer cuatrimestre del curso 2018-2019 se ha planificado el proyecto y se ha empezado a implantar durante el segundo cuatrimestre de éste.

Los objetivos específicos son: 
- Diseñar actividades específicas en la asignatura de "Estadística" de forma que se integre de forma transversal con los objetivos de aprendizaje de la asignatura de "Energía y desarrollo sostenible"

- Observar los resultados obtenidos de la implantación de esta metodología en el primer curso del grado (aceptación de los alumnos,

En los siguientes apartados se presenta el contexto y el desarrollado de la innovación, la metodología y los primeros resultados obtenidos. Por último, se incluyen las conclusiones del estudio.

\section{Desarrollo de la innovación}

Las asignaturas en las cuales se va aplicar el aprendizaje basado en proyectos son la asignatura de "Estadística" del primer curso y la asignatura de "Energía y Desarrollo Sostenible" del segundo curso del Grado de Ingeniero de la Energía. Ambas asignaturas son obligatorias y, por tanto, todos los alumnos matriculados en el grado deben cursarla. El tamaño medio de grupo durante los últimos años se ha mantenido relativamente constante, entre 75 y 85 alumnos.

En los últimos años, se ha detectado que los alumnos presentan problemas a la hora de aplicar los conceptos teóricos vistos en el primer curso en la asignatura de Estadística con la aplicabilidad práctica de problemas reales presentados en la asignatura de $2^{\circ}$ curso. Este problema implica perder oportunidades de sinergia entre las distintas asignaturas para trabajar de forma más efectiva algunas competencias. En este contexto, el objetivo principal de este proyecto es el de desarrollar un proyecto que integre las competencias específicas y transversales de estas asignaturas para que el alumno pueda aplicar de forma práctica los conceptos en un contexto real. El alumno al mismo tiempo que utiliza las herramientas estadísticas desarrolla dos capacidades: la de calcular y la de trabajar en un proyecto del sector energético.

Como se ha mencionado anteriormente, el desarrollo del proyecto se encuentra en el primer año de ejecución, por tanto, según lo planificado, se está desarrollando la metodología en la asignatura del primer curso. La asignatura de estadística tiene según su guía docente 0,8 créditos de prácticas de informática, planificadas en cuatro sesiones prácticas de 2 horas de duración. La innovación se está llevando a cabo durante las sesiones de prácticas en las que el alumno tendrá que aplicar en cada una de ellas la estadística para resolver problemas relacionados con la energía. Estas sesiones aplicadas servirán para que posteriormente puedan utilizar la estadística como una herramienta en la toma de decisiones en su campo.

Uno de los problemas que apareció en las primeras reuniones fue el de elegir la temática del proyecto. Por tanto, el primer paso consistió en fijar la temática de las sesiones prácticas. Para ello se elaboró un cuestionario a los alumnos con el objetivo de observar las preferencias que tienen actualmente los alumnos del GIE. Este cuestionario se pasó a los 86 alumnos matriculados en el curso 2018/2019 de la asignatura de estadística. Los resultados obtenidos se muestran en las Figura 1 y Figura 2. La Figura 1 muestra el interés de los matriculados encuestados en los dos tipos de energía: las energías renovables y las no renovables. La 
Figura 2 muestra el interés por parte de los alumnos encuestados en desarrollar un proyecto relacionado con diferentes fuentes de energía.

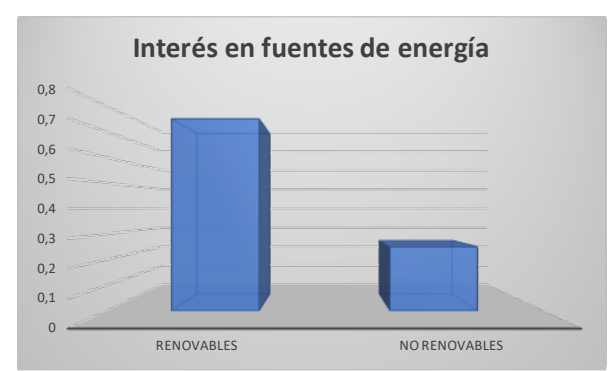

Fig. 1 Interés por el tipo de energía

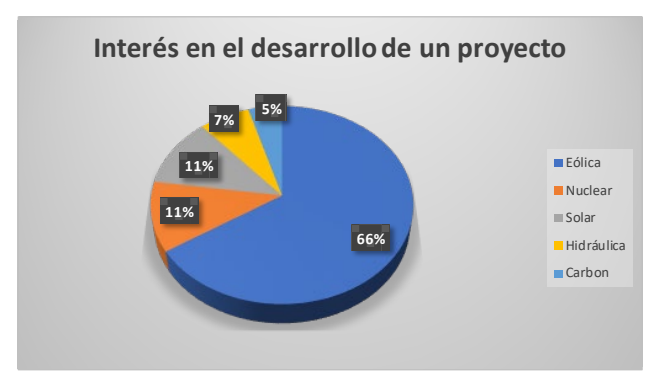

Fig. 2 Interés en el tema para el desarrollo de un proyecto académico

Tras analizar los resultados de dicha encuesta, se obtuvieron las siguientes conclusiones:

- $\quad$ El 75\% de los alumnos encuestados están interesados en el campo de las energías renovables.

- El 81\% de los alumnos considera la estadística a nivel teórico como una herramienta muy útil en la toma de decisiones, siendo una técnica capaz de predecir el consumo eléctrico, faltándoles una perspectiva más aplicada.

- Además, al 66\% de los alumnos le gustaría desarrollar a lo largo de su trayectoria académica un proyecto relacionado con la energía eólica.

En este contexto, mediante los resultados obtenidos con la encuesta, junto con el conocimiento previo por parte de los profesores de la asignatura de "Energía y Desarrollo Sostenible", se decidió realizar el proyecto en el campo de la energía eólica, para que los alumnos se motiven utilizando datos reales aplicados a su campo profesional y fomentar la participación activa en el aula.

Tras la elección del tema conductor del proyecto y después de varias reuniones tanto con los profesores del DEIOAC como del DIQN, se propusieron las siguientes sesiones prácticas para la asignatura de "Estadística":

- Practica 1. Análisis estadístico descriptivo de la velocidad del viento y la potencia generada por un aerogenerador.

- Práctica 2. Estudiar la curva de potencia de un aerogenerador desde el punto de vista cualitativo.

- Práctica 3. Caracterización de la distribución Weibull de velocidades de viento a partir de los datos recogidos en el sistema de adquisición de datos de un parque eólico.

- Práctica 4. Comparativa entre diferentes localizaciones para la toma de decisiones a la hora de instalar un parque eólico utilizando para ello la inferencia estadística. 
Esta primera etapa de planificación de actividades ha supuesto un gran esfuerzo de coordinación entre las dos asignaturas para utilizar la misma nomenclatura y trabajar en los mismos proyectos desde el principio. Además, se han tenido que optimizar los recursos utilizados en el aula, tanto los contenidos como las herramientas utilizadas para mejorar la sinergia e integración de los conceptos básicos de estadística en la asignatura de segundo.

Hoy en día queda pendiente trabajar de forma explícita en el aprendizaje basado en proyectos en la asignatura de $2^{\circ}$ curso, aunque sí que se han realizado pequeñas incursiones en esta metodología en las sesiones de prácticas, recibiendo en estos casos una realimentación muy positiva por parte de alumnos.

\section{Resultados}

A continuación, se muestran los resultados obtenidos en el desarrollo de esta innovación. Por una parte, se han desarrollado resultados directos de la coordinación entre asignaturas (unificación y clarificación de conceptos, actividades diseñadas, tablas de indicadores y rúbricas generadas) y, por otro lado, resultados obtenidos a partir de la innovación midiendo el impacto en el aula en los alumnos de primer curso. Cabe señalar, que los resultados mostrados hacen referencia a la planificación y desarrollo de la metodología y que, al finalizar el presente curso académico (2018-2019) con los resultados de los alumnos se llevará a cabo el análisis global de la implantación en el primer curso del grado.

\subsection{Resultados directos de la coordinación}

Los resultados obtenidos de las reuniones de coordinación y planificación realizadas durante el desarrollo de esta primera etapa de la innovación han sido uno de los más importantes ya que han permitido establecer las bases del entendimiento entre los profesores unificando y clarificando conceptos. Además, de determinar las limitaciones y necesidades de los alumnos en las asignaturas de ambos cursos. Estas reuniones han servido para planificar el proyecto y establecer las bases para las distintas competencias a considerar en ambas asignaturas.

Además, se han desarrollado las actividades de la asignatura de "Estadística", con el objetivo de mejorar las sinergias entre las dos asignaturas. Para cada una de las actividades planteadas en el proyecto se ha desarrollado una ficha descriptiva con la estructura del fragmento mostrado en la Tabla 1. Como ejemplo, en la asignatura de "Energía y desarrollo sostenible" de segundo los alumnos tendrán que estudiar, entre otros factores, la velocidad del viento para determinar el recurso eólico y la potencia generada en un parque eólico cuando tengan que diseñar un parque eólico. Por tanto, es importante que el alumno cuando llegue a la asignatura de segundo entienda y sepa caracterizar la función de densidad y de probabilidad de la velocidad del viento, para ello se plantea la práctica 3, cuyos resultados de aprendizaje se pueden consultar en la Tabla 1.

Tabla 1. Ejemplo de ficha de actividad (fragmento). 


\begin{tabular}{|c|c|}
\hline Actividad. & $\begin{array}{l}\text { Caracterización de la distribución Weibull de velocidades de } \\
\text { viento a partir de los datos recogidos en el sistema de adquisición } \\
\text { de datos de un parque eólico. }\end{array}$ \\
\hline Asignaturas. & "Estadística" \\
\hline $\begin{array}{l}\text { Competencias } \\
\text { transversales. }\end{array}$ & $\begin{array}{l}\text { (01) Comprensión e integración. } \\
\text { (02) Aplicación y pensamiento práctico. } \\
\text { (11) Aprendizaje Permanente. }\end{array}$ \\
\hline Descripción. & 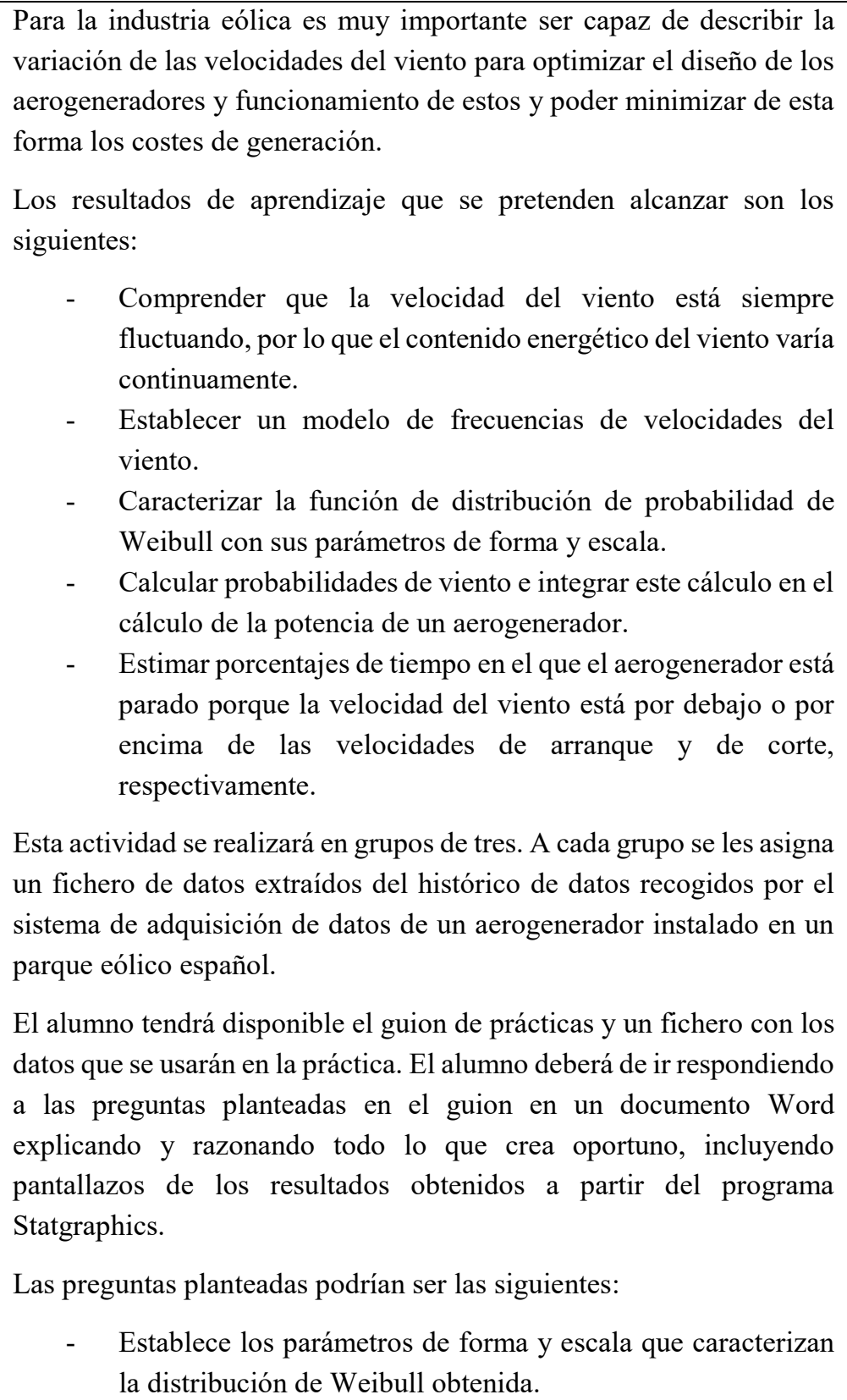 \\
\hline
\end{tabular}




\begin{tabular}{|c|c|}
\hline & $\begin{array}{l}\text { - Define la función de densidad y de distribución de Weibull } \\
\text { para la velocidad de viento. } \\
\text { - } \quad \text { Calcula probabilidades a partir de la función de distribución. } \\
\text { - Aplica la función de distribución de Weibull para calcular } \\
\text { potencia de un aerogenerador. Curva de Potencia. }\end{array}$ \\
\hline
\end{tabular}

Además, se han generado rúbricas para evaluar la adquisición de diversas competencias transversales de estas actividades. De momento, únicamente se han realizado para la asignatura de estadística adaptando en cada caso el nivel de dominio y enfoque de la actividad.

\subsection{Resultados en aula}

El segundo tipo de resultados (impacto en el alumnado) se mide en cuanto a generación de evidencias del trabajo tras aplicar esta innovación docente.

En este caso, los resultados obtenidos aún no son del todo visibles, ya que es el primer año en el que se lleva a cabo esta metodología enmarcada dentro de un proyecto de innovación docente y todavía no se dispone de retroalimentación por parte de los alumnos. Aún así, se ha podido observar que el alumnado se muestra más receptivo en el aula y asimilan mejor los conceptos teóricos al poder relacionarlos con problemas reales. Además, el alumno está tomando un papel más activo en el proceso de aprendizaje encontrándose más motivados y mejorando la relación y la participación en la comunicación con el profesor. Cabe destacar que todas estas evidencias se podrán justificar en un futuro analizando los resultados de las encuestas de evaluación de la docencia. En cualquier caso, los profesores han elaborado un cuestionario de satisfacción que se pasará al final del semestre con el objetivo de tener una idea aproximada del nivel de aceptación por parte del alumno de la metodología seguida en las sesiones prácticas. Se trata de preguntas "informales" como las siguientes:

- ¿Te han parecido útil estas actividades?

- ¿Te ha cambiado la percepción del uso de la "Estadística” en el campo de la energía?

- ¿Qué actividad/Práctica te ha gustado más?

\section{Conclusiones}

Este trabajo se encuentra enmarcado dentro de un proyecto docente en el cual se pretende desarrollar y establecer metodologías, definir técnicas de evaluación apropiadas de evaluación centradas en el aprendizaje basado en proyectos en la asignatura de "Energía y Desarrollo Sostenible", potenciando el uso de los conceptos aprendidos en la asignatura de "Estadística" en el Grado de Ingeniero de la Energía. Este proyecto se encuentra en el primer año de ejecución, por tanto, en este trabajo se muestran únicamente el planteamiento inicial correspondiente con las actividades desarrolladas para la asignatura de "Estadística".

Aunque actualmente no se tenga mucha retroalimentación por parte de los alumnos, las primeras impresiones por parte del alumnado son muy positivas, acogiendo de forma activa este tipo de metodologías. Aunque sea pronto, ya se vislumbra que los alumnos van alcanzando los resultados de aprendizaje establecidos con un nivel mayor que el que daban

(c)) BY-NC-ND 2019, Universitat Politècnica de València 
las prácticas de laboratorio tradicionales. Esta metodología está permitiendo al mismo tiempo desarrollar y evaluar tanto las competencias específicas de la asignatura como desarrollar las competencias transversales comprensión e integración y aplicación y pensamiento práctico.

En el próximo año se pretende llevar a cabo esta metodología de forma más profunda para la asignatura de "Energía y Desarrollo Sostenible" y poder obtener conclusiones de forma más global.

Debido a que se propone trabajar con alumnos de distintos cursos y con distinto grado de madurez académica, no se esperan resultados de manera inmediata, ya que se necesitará al menos tres años para tener una retroalimentación fiable de alumnos que hayan seguido el itinerario de las asignaturas objeto de esta innovación docente.

\section{Agradecimientos}

Este trabajo se ha realizado en el marco del Proyecto de Innovación y Mejora Educativa, PIME Curso 2018-2019 "Coordinación de competencias transversales en asignaturas de ámbito nuclear en el Grado de Ingeniero de la Energía”, Referencia B16, del Vicerrectorado de Estudios, Calidad y Acreditación de la Universitat Politècnica de València.

\section{Referencias}

MARIMOM, M. (2013). Colaborar para aprender. En G. Bautista y A. Es $\neg$ cofet (Comp.). Enseñar y aprender en la universidad. Claves y retos para la mejora (pp. 47-72).

GUERRERO, E., CALERO, J. (2013) "El aprendizaje basado en proyectos como base metodológica en el grado de Educación Social” Educación social. Revista de Intervención Socioeducativa, 53, p. 7391

HUFF, J. L., ZOLTOWSKI, C. B. AND OAKES, W. C. (2016), Preparing Engineers for the Workplace through Service Learning: Perceptions of EPICS Alumni. Journal of Engineering Education. 105,4369.

GARCIA, J., PEREZ, J. (2018) Aprendizaje basado en proyectos: método para el diseño de Actividades. Revista Tecnología, Ciencia e Innovación, CEF, núm. 10 (mayo-agosto 2018, pp. 37-63

CUIÑAS I., MARIÑO-ESPIÑEIRA P., FERNANDEZ-IGLESIAS M., CAEIRO, M., COSTAMONTENEGRO E., DIAZ-OTERO F. (2016). Evaluación de competencias con metodologías de aprendizaje basado en proyectos. Congreso INRED 2016 